\title{
Correction to: Economics of Preventing, Screening, and Treating Oral Cancers
}

\author{
Sujha Subramanian and K. Thankappan
}

\section{Correction to: \\ Chapter 9 in: M.A. Kuriakose (ed.), Contemporary Oral Oncology, https://doi.org/10.1007/978-3-319-43857-3_9}

The original version of this chapter did not state that permission was received to reuse text from the following source:

- Subramanian S. (2013) Cost-Effectiveness of Screening for and Early Diagnosis of Breast and Gynecological Cancers in Low-Income Countries. In: Shetty M. (eds) Breast and Gynecological Cancers. Springer, New York, NY

The authors have obtained retrospective permission to use this source.

In addition, the authors wish to cite the following source as it was also used to write this chapter:

- Sankaranarayanan R, Ramadas K, Amarasinghe H, Subramanian S, Johnson N. "Oral Cancer: Prevention, Early Detection, and Treatment”. In: Disease Control Priorities (third edition): Volume 3, Cancer, edited by H. Gelband, P. Jha, R. Sankaranarayanan, S. Horton. Washington, DC: World Bank.

Furthermore, the chapter was published with an incorrect spelling of one of the authors' names as K. Krishnakumar whereas it has been K. Thankappan.

The updated version of this chapter can be found at https://doi.org/10.1007/978-3-319-43857-3_9 